\title{
On Mann's Method with Viscosity for Nonexpansive and Nonspreading Mappings in Hilbert Spaces
}

\author{
Nawab Hussain, ${ }^{1}$ Giuseppe Marino, ${ }^{1,2}$ and Afrah A. N. Abdou ${ }^{1}$ \\ ${ }^{1}$ Department of Mathematics, King Abdulaziz University, P.O. Box 80203, Jeddah 21589, Saudi Arabia \\ ${ }^{2}$ Dipartimento di Matematica, Universitá della Calabria, 87036 Arcavacata di Rende, Italy \\ Correspondence should be addressed to Nawab Hussain; nhusain@kau.edu.sa
}

Received 9 May 2014; Accepted 8 June 2014; Published 9 July 2014

Academic Editor: Jen-Chih Yao

Copyright (c) 2014 Nawab Hussain et al. This is an open access article distributed under the Creative Commons Attribution License, which permits unrestricted use, distribution, and reproduction in any medium, provided the original work is properly cited.

In the setting of Hilbert spaces, inspired by Iemoto and Takahashi (2009), we study a Mann's method with viscosity to approximate strongly (common) fixed points of a nonexpansive mapping and a nonspreading mapping. A crucial tool in our results is the nonspreading-average type mapping.

\section{Introduction}

Let $H$ be a real Hilbert space with inner product $\langle\cdot, \cdot\rangle$ that induces the norm $\|\cdot\|=\sqrt{\langle\cdot, \cdot\rangle}$. Let $T: \operatorname{Dom}(T) \rightarrow H$ be a mapping. We denote by $\operatorname{Fix}(T)$ the set of fixed points of $T$, $\operatorname{Fix}(T)=\{x \in \operatorname{Dom}(T): T x=x\}$.

A mapping $T$ is said to be

(i) nonexpansive [1] (1967) if $\|T x-T y\| \leq\|x-y\|$ for all $x, y \in \operatorname{Dom}(T)$

(ii) firmly nonexpansive [1] (1967) if $\langle x-y, T x-T y\rangle \geq$ $\|T x-T y\|^{2}$ for all $x, y \in \operatorname{Dom}(T)$;

(iii) firmly type nonexpansive [2] (2009) if

$$
\|T x-T y\|^{2} \leq\|x-y\|^{2}-k\|(I-T) x-(I-T) y\|^{2}
$$

for all $x, y \in \operatorname{Dom}(T)$ for all $x, y \in \operatorname{Dom}(T)$;

(iv) strongly nonexpansive [3] (1977) if $T$ is nonexpansive and $\left(x_{n}\right)_{n \in \mathbb{N}},\left(y_{n}\right)_{n \in \mathbb{N}} \subset \operatorname{Dom}(T),\left\|x_{n}-y_{n}\right\|$ bounded and $\left(\left\|x_{n}-y_{n}\right\|-\left\|T x_{n}-T y_{n}\right\|\right) \rightarrow 0$ then $\|(I-T) x_{n}-$ $(I-T) y_{n} \| \rightarrow 0$ for all $x, y \in \operatorname{Dom}(T)$;

(v) nonspreading [4] (2008) if $2\|T x-T y\|^{2} \leq\|T x-y\|^{2}$ $+\|x-T y\|^{2}$ for all $x, y \in \operatorname{Dom}(T)$; (vi) $k$-strict nonspreading [5] (2011) if

$$
\begin{aligned}
\|T x-T y\|^{2} \leq & \|x-y\|^{2}+2\langle x-T x, y-T y\rangle \\
& +k\|(I-T) x-(I-T) y\|^{2} ;
\end{aligned}
$$

(vii) quasi-nonexpansive if $\|T x-y\| \leq\|x-y\|$ for all $x \in$ $\operatorname{Dom}(T)$ and for all $y \in \operatorname{Fix}(T)$.

Of course,

firmly nonexpansive $\Rightarrow$ firmly type nonexpansive $\Rightarrow$ strongly nonexpansive $\Rightarrow$ nonexpansive $\Rightarrow$ quasi-nonexpansive $\Leftarrow$ nonspreading $\Leftarrow k$-strict pseudononspreading.

If $C$ is a nonempty, closed, and convex subset of $H$, we denote by $P_{C}: H \rightarrow C$ the metric projection on $C$; that is, for any $x \in H, P_{C} x$ is the unique element in $C$ such that

$$
\left\|x-P_{C} x\right\| \leq\|x-y\|, \quad \forall y \in C .
$$

It is well known (see [1]) that $P_{C}$ is a firmly nonexpansive mapping and that $P_{C}$ is characterized by the variational inequality

$$
\left\langle P_{C} x-x, z-P_{C} x\right\rangle \geq 0, \quad \forall z \in C .
$$

The firm nonexpansivity has many equivalent formulations.

Theorem 1. Let $T: C \rightarrow C$ a mapping. There are equivalents. 
(1) $T$ is firmly nonexpansive; that is, $\langle x-y, T x-T y\rangle \geq$ $\|T x-T y\|^{2}$ for all $x, y \in \operatorname{Dom}(T)$.

(2) For each $x, y \in C$, the convex function $\Phi_{x, y}:[0,1] \rightarrow$ $\mathbb{R}$ defined by

$$
\Phi_{x, y}(t)=\|(1-t)(x-y)+t(T x-T y)\|
$$

is nonincreasing on $[0,1]$.

(3) The mapping $(2 t-I)$ is nonexpansive.

(4) $T=(1 / 2)(I+N)$ with $N$ is nonexpansive.

(5) $\|T x-T y\| \leq \Phi_{x, y}(t)$ for all $t \in[0,1]$.

(6) The mapping $(I-T)$ is firmly nonexpansive.

(7) $\|T x-T y\|^{2} \leq\|x-y\|^{2}-\|(I-t) x-(I-T) y\|^{2}$ holds.

(8) One has $2\|T x-T y\|^{2}+\|T x-x\|^{2}+\|T y-y\|^{2} \leq$ $\|T x-y\|^{2}+\|T y-x\|^{2}$.

Proof. The equivalences (1) to (5) are proved in [6]. The equivalences (1) and (6) are proved in [7]. Let us prove that (1) is equivalent to (8):

$$
\begin{aligned}
2\|T x-T y\|^{2}+\|T x-x\|^{2}+\|T y-y\|^{2} \\
\leq\|T x-y\|^{2}+\|T y-x\|^{2} \\
\Longleftrightarrow 2\|T x-T y\|^{2}-2\langle T x, x\rangle-2\langle T y, y\rangle+\|T x\|^{2}+\|y\|^{2} \\
\leq\|T x\|^{2}+\|y\|^{2}+\|T y\|^{2} \\
\quad+\|x\|^{2}-2\langle T x, y\rangle-2\langle T y, x\rangle \\
\Longleftrightarrow 2\|T x-T y\|^{2}-2\langle T x, x\rangle-2\langle T y, y\rangle \\
\leq-2\langle T x, y\rangle-2\langle T y, x\rangle \\
\Longleftrightarrow 2\|T x-T y\|^{2} \\
\leq 2\langle T x, x\rangle+2\langle T y, y\rangle-2\langle T x, y\rangle-2\langle T y, x\rangle \\
=2\langle T x, x-y\rangle-2\langle T y, x-y\rangle=2\langle T x-T y, x-y\rangle \\
\Longleftrightarrow\|T x-T y\|^{2} \leq\langle T x-T y, x-y\rangle .
\end{aligned}
$$

Finally, we prove that (1) is equivalent to (7). $(I-T)$ is firmly nonexpansive $\Leftrightarrow\langle x-y,(I-T) x-(I-T) y\rangle$ $\geq\|(I-T) x-(I-T) y\|^{2} \Leftrightarrow\|(I-T) x-(I-T) y\|^{2} \leq$ $\|x-y\|^{2}-\langle x-y, T x-T y\rangle \leq\|x-y\|^{2}-\|T x-T y\|^{2}$.

Two important classes of mappings containing the firmly nonexpansive mappings are the average mappings and the nonspreading mappings.

After [7], we say that $T$ is an nonexpansive-average mappings if

$$
T=(1-\alpha) I+\alpha N
$$

for some $\alpha \in(0,1)$, and $N$ is a nonexpansive mapping.
Definition 2. Let $\mathfrak{M}$ be a class of mappings. One says that $T$ is a $\mathfrak{M}$-average mapping if

$$
T=N_{\alpha}=(1-\alpha) I+\alpha N
$$

for some $\alpha \in(0,1)$ where $N$ is a mapping belonging to the class $\mathfrak{M}$.

Of course $\operatorname{Fix}\left(N_{\alpha}\right)=\operatorname{Fix}(N)$.

The nonexpansive-average mapping regularizes a nonexpansive mapping $N$ according to the celebrated Schaefer's result [8].

Theorem 3. Any orbit $\left(N_{\alpha}^{k} x\right)_{k \in \mathbb{N}}$ of a nonexpansive-average mapping $N_{\alpha}=(1-\alpha) I-\alpha N$ converges weakly to a fixed point of $N$ whenever such points exist.

Here we are interested in nonspreading and non-spreading-average mappings.

Theorem 4. Let $S: C \rightarrow C$ be a mapping. The following are equivalent.

(1) $S$ is nonspreading; that is, $2\|T x-T y\|^{2} \leq\|T x-y\|^{2}+$ $\|x-T y\|^{2}$

(2) One has $\|S x-S y\|^{2} \leq\|x-y\|^{2}+2\langle x-S x, y-S y\rangle$;

(3) $\|S x-S y\|^{2} \leq\|x-y\|^{2}+\|x-S x\|^{2}+\|y-S y\|^{2}-$ $\|(I-S) x-(I-S) y\|^{2}$.

Moreover, let $S$ be a nonspreading mapping. Then

(a) $\operatorname{Fix}(S)$ is closed and convex;

(b) $(I-S)$ is demiclosed;

(c) One has $\|(I-S) x-(I-S) y\|^{2} \leq\langle x-y,(I-S) x-(I-$ S) $y\rangle+(1 / 2)\|(I-S) x\|^{2}+(1 / 2)\|(I-S) y\|^{2}$.

If $S_{\omega}=(1-\omega) I+\omega S$ is a nonspreading-average mapping, then one has the following.

(i)

$$
\begin{aligned}
\left\|S_{\omega} x-S_{\omega} y\right\|^{2} \leq & \|x-y\|^{2}+\frac{2}{\omega}\left\langle x-S_{\omega} x, y-S_{\omega} y\right\rangle \\
& -(1-\omega)\left\|\left(x-S_{\omega} x\right)-\left(y-S_{\omega} y\right)\right\|^{2} .
\end{aligned}
$$

In particular $S_{\omega}$ is quasi firmly nonexpansive; that is,

$$
\left\|S_{\omega} x-q\right\|^{2} \leq\|x-q\|^{2}-(1-\omega)\left\|x-S_{\omega} x\right\|^{2} .
$$

(ii) $(\omega / 2)\|x-S x\|^{2} \leq\left\langle x-S_{\omega} x, x-q\right\rangle$, for all $q \in \operatorname{Fix}(S)$.

Proof. The equivalence of (1) and (2) is proved in Lemma 3.2 of [9].

The equivalence of (1) and (3) follows by the fact that

$$
\begin{aligned}
2\left\langle x-S_{\omega} x, x-q\right\rangle= & -\|(x-S x)-(y-S y)\|^{2} \\
& +\|x-S x\|^{2}+\|y-S y\|^{2} .
\end{aligned}
$$


The item (a) is proved in [4], while (b) and (c) are proved in [9].

The item (i) is proved in Theorem 3.1 of [5].

Now we prove (ii). Since

$$
\begin{aligned}
\left\langle x-S_{\omega} x, x-q\right\rangle & =\langle x-(1-\omega) x-S x, x-q\rangle \\
& =\omega\langle x-S x, x-q\rangle
\end{aligned}
$$

thus we need to show that

$$
\frac{1}{2}\|x-S x\|^{2} \leq\langle x-S x, x-q\rangle .
$$

This follows by quasi-nonexpansivity of $S$. Indeed

$$
\begin{array}{r}
\frac{1}{2}\|x-S x\|^{2}= \\
=\frac{1}{2}\|(x-q)+(q-S x)\|^{2} \\
=\frac{1}{2}\left[\|x-q\|^{2}+\|q-S x\|^{2}\right. \\
+2\langle x-q, q-S x\rangle]
\end{array}
$$

(by the quasi-nonexpansivity of $S$ )

$$
\begin{aligned}
\leq & \|x-q\|^{2} \\
& +\langle x-q, q-S x\rangle \\
= & \langle x-q, x-q\rangle \\
& +\langle x-q, q-S x\rangle \\
= & \langle x-q, x-S x\rangle .
\end{aligned}
$$

Recently, Song and Chai [2] in the general setting of Banach spaces obtained strong convergence of Halpern's iteration

$$
x_{n+1}=\alpha_{n} u+\left(1-\alpha_{n}\right) T x_{n}
$$

for firmly type nonexpansive mapping T. (Saejung in [10] noted that their proof seems to be questionable, but the result is true as a consequence of a more general result proved in [10]). Indeed, in [10] is proved the strong convergence of Halpern iteration for strongly nonexpansive mappings (and it is easy to see that the class of strongly nonexpansive mappings contains the class of firmly type nonexpansive mappings).

Osilike and Isiogugu [5] studied the Halpern iteration for $k$-strict pseudo-non-spreading mappings. They showed that if one considers the $k$-strict pseudo-non-spreading-average mapping, then Halpern's iteration converges strongly to a fixed point of such a mapping.

On the other hand, Iemoto and Takahashi [9] approximated weakly fixed points of nonexpansive mappings and/or a nonspreading mapping in a Hilbert space using Moudafi's iteration scheme [11]. Specifically, they proved the following result.

Theorem 5. Let $H$ be a Hilbert space and let $C$ be a nonempty closed convex subset of $H$. Let $S$ be a nonspreading mapping on
$C$ into itself and let $T$ be a nonexpansive mappings on $C$ into itself such that $\operatorname{Fix}(T) \cap \operatorname{Fix}(S) \neq \emptyset$. Define a sequence $\left(x_{n}\right)_{n \in \mathbb{N}}$ as follows:

$$
\begin{gathered}
x_{1} \in C, \\
x_{n+1}=\left(1-\alpha_{n}\right) x_{n}+\alpha_{n}\left[\beta_{n} S x_{n}+\left(1-\beta_{n}\right) T x_{n}\right]
\end{gathered}
$$

for all $n \in \mathbb{N}$, where $\left(\alpha_{n}\right)_{n \in \mathbb{N}},\left(\beta_{n}\right)_{n \in \mathbb{N}}$, are in $[0,1]$. Then the following holds.

(I) if $\lim \inf \alpha_{n}\left(1-\alpha_{n}\right)>0$ and $\sum\left(1-\beta_{n}\right)<\infty$, then $\left(x_{n}\right)_{n}$ weakly converges to $v \in \operatorname{Fix}(S)$.

(II) if $\sum \alpha_{n}\left(1-\alpha_{n}\right)=\infty$ and $\sum \beta_{n}<\infty$, then $\left(x_{n}\right)_{n}$ weakly converges to $v \in \operatorname{Fix}(T)$.

(III) if $\lim \inf \alpha_{n}\left(1-\alpha_{n}\right)>0$ and $\lim \inf \beta_{n}\left(1-\beta_{n}\right)>0$, then $\left(x_{n}\right)_{n}$ weakly converges to $v \in \operatorname{Fix}(T) \cap \operatorname{Fix}(S)$.

In [12], the authors obtained strong convergence for the Halpern method by using type average mappings, with assumptions on the coefficients very similar to Theorem 5.

So one can ask if this result holds for Moudafi's viscosity method [13]. We cannot take advantage of using the above positive results on Halpern's iteration and invoke Suzuki's result [14] that affirms that Halpern's approximation convergence implies Moudafi's viscosity approximation convergence. Indeed, as proved by Suzuki, this is true for nonexpansive mappings not for nonspreading mappings.

In spite of this we obtain the affirmative answer in our main result.

Our proofs took inspiration by $[5,12,15,16]$. Related papers in which there are not nonspreading but other types of mappings or semigroups of nonexpansive mappings are [1723].

\section{Main Results}

In this section, we always will assume the following.

(i) $H$ is a Hilbert space.

(ii) $C$ is a closed and convex subset of $H$.

(iii) $T: C \rightarrow C$ is a nonexpansive mapping.

(iv) $T_{\omega}: C \rightarrow C$ is an average mapping of $T, T_{\omega}=(1-$ $\omega) I+\omega T$.

(v) $S: C \rightarrow C$ is a nonspreading mapping.

(vi) $S_{\omega}: C \rightarrow C$ is a nonspreading-average mapping of $S$, $S_{\omega}=(1-\omega) I+\omega S$.

(vii) $U_{n}: C \rightarrow C$ is a convex combination of $T_{\omega}$ and $S_{\omega}$, $U_{n}=\left(1-\beta_{n}\right) S_{\omega}+\beta_{n} T_{\omega}$.

(viii) $\operatorname{Fix}(T) \cap \operatorname{Fix}(S) \neq \emptyset$.

(ix) $f: C \rightarrow C$ is a $\rho$-contraction; that is, $\|f(x)-f(y)\| \leq$ $\rho\|x-y\|, 0<\rho<1$.

(x) $\left(\alpha_{n}\right)_{n \in \mathbb{N}} \subset[0,1]$ is a real sequence satisfying $\alpha_{n} \rightarrow 0$ and $\sum_{n=1}^{\infty} \alpha_{n}=\infty$.

(xi) $O(1)$ denote any bounded real sequence (so $O(1)+$ $O(1)=O(1))$. 

result.

The following lemmas are the keys to obtain our main

Lemma 6 (see [24]). Assume that $\left(a_{n}\right)_{n \in \mathbb{N}}$ is a sequence of nonnegative numbers such that

$$
a_{n+1} \leq\left(1-\gamma_{n}\right) a_{n}+\gamma_{n} \delta_{n}+\sigma_{n}, \quad n \geq 0,
$$

where $\left(\gamma_{n}\right)_{n}$ is a sequence in $(0,1)$ and $\left(\delta_{n}\right)_{n}$ is a sequence in $\mathbb{R}$ and $\left(\sigma_{n}\right)_{n} \subset \mathbb{R}^{+}$such that,

(1) $\sum_{n=1}^{\infty} \gamma_{n}=\infty$;

(2) $\lim \sup _{n \rightarrow \infty} \delta_{n} \leq 0$ and $\sum_{n=1}^{\infty}\left|\sigma_{n}\right|<\infty$.

Then, $\lim _{n \rightarrow \infty} a_{n}=0$.

Lemma 7. Let $\left(x_{n}\right)_{n}$ be the sequence defined by

$$
x_{n+1}=\alpha_{n} f\left(x_{n}\right)+\left(1-\alpha_{n}\right) U_{n} x_{n} .
$$

Then, (i) $U_{n}$ is quasi nonexpansive; (ii) $\left(x_{n}\right)_{n},\left(S x_{n}\right)_{n},\left(T x_{n}\right)_{n}$, $\left(S_{\omega} x_{n}\right)_{n},\left(T_{\omega} x_{n}\right)_{n}$, and $\left(U_{n} x_{n}\right)_{n}$ are bounded sequences.

Proof. (i) Any convex combination of quasi nonexpansive mappings is, in turn, quasi nonexpansive. So is $U_{n}$, since $T_{\omega}$ and $S_{\omega}$ are quasi nonexpansive (see Theorem 4 , (i)).

(ii) We see that the boundedness of $\left(x_{n}\right)_{n}$ follows by the quasi nonexpansivity of $U_{n}$. For this let $q \in \operatorname{Fix}(T) \cap \operatorname{Fix}(S)$. Then

$$
\begin{aligned}
\left\|x_{n+1}-q\right\| \leq & \alpha_{n}\left\|f\left(x_{n}\right)-q\right\|+\left(1-\alpha_{n}\right)\left\|U_{n} x_{n}-q\right\| \\
\leq & \alpha_{n}\left\|f\left(x_{n}\right)-f(q)\right\|+\alpha_{n}\|f(q)-q\| \\
& +\left(1-\alpha_{n}\right)\left\|U_{n} x_{n}-q\right\| \\
\leq & \alpha_{n} \rho\left\|x_{n}-z\right\|+\alpha_{n}\|f(q)-q\| \\
& +\left(1-\alpha_{n}\right)\left\|x_{n}-q\right\| \\
\leq & \left(1-\alpha_{n}(1-\rho)\right)\left\|x_{n}-q\right\|+\alpha_{n}\|f(q)-q\| \\
= & \left(1-\alpha_{n}(1-\rho)\right)\left\|x_{n}-q\right\| \\
& +\alpha_{n}(1-\rho) \frac{\|f(q)-q\|}{1-\rho} \\
& \quad(\operatorname{byc} \operatorname{convexity~of~}\|\cdot\|) \\
\leq & \max \left\{\left\|x_{n}-q\right\|, \frac{\|f(q)-q\|}{1-\rho}\right\} \Longrightarrow\left\|x_{n+1}-q\right\| \\
\leq & \max \left\{\left\|x_{1}-q\right\|, \frac{\|f(q)-q\|}{1-\rho}\right\} .
\end{aligned}
$$

The boundedness of $\left(x_{n}\right)_{n}$ is proved. The boundedness of the other sequences in (ii) follows by this last (since $\operatorname{Fix}(T) \cap$ $\operatorname{Fix}(S) \neq \emptyset)$.
Lemma 8. Let $\left(y_{n}\right)_{n}$ be a bounded sequence in $C$. Then one has the following.

(i) If $\left\|y_{n}-T y_{n}\right\| \rightarrow 0$, then

$$
\lim \sup \left\langle(I-f) \bar{p}, y_{n}-\bar{p}\right\rangle \geq 0,
$$

where $\bar{p}=P_{\operatorname{Fix}(T)} f(\bar{p})$ is the unique point in $\operatorname{Fix}(T)$ that satisfies the variational inequality

$$
\langle(I-f) \bar{p}, x-\bar{p}\rangle \geq 0, \quad \forall x \in \operatorname{Fix}(T) .
$$

(ii) If $\left\|y_{n}-S y_{n}\right\| \rightarrow 0$, then

$$
\lim \sup \left\langle(I-f) \tilde{p}, y_{n}-\tilde{p}\right\rangle \geq 0,
$$

where $\tilde{p}=P_{\operatorname{Fix}(S)} f(\tilde{p})$ is the unique point in $\operatorname{Fix}(S)$ that satisfies the variational inequality

$$
\langle(I-f) \tilde{p}, x-\tilde{p}\rangle \geq 0, \quad \forall x \in \operatorname{Fix}(T) .
$$

(iii) If both $\left\|y_{n}-S y_{n}\right\| \rightarrow 0$ and $\left\|y_{n}-T y_{n}\right\| \rightarrow 0$, then

$$
\lim \sup \left\langle(I-f) p_{0}, y_{n}-p_{0}\right\rangle \geq 0,
$$

where $p_{0}=P_{\mathrm{Fix}(T) \cap \operatorname{Fix}(S)} f\left(p_{0}\right)$ is the unique point in $\operatorname{Fix}(T) \cap \operatorname{Fix}(S)$ that satisfies the variational inequality

$$
\left\langle(I-f) p_{0}, x-p_{0}\right\rangle \geq 0, \quad \forall x \in \operatorname{Fix}(T) \cap \operatorname{Fix}(S) .
$$

Proof. (i) Let $\bar{p}$ satisfy (21). Let $\left(y_{n_{k}}\right)$ be a subsequence of $\left(y_{n}\right)$ for which

$$
\limsup _{n}\left\langle(I-f) \bar{p}, y_{n}-\bar{p}\right\rangle=\lim _{k}\left\langle(I-f) \bar{p}, y_{n_{k}}-\bar{p}\right\rangle .
$$

Select a subsequence $\left(y_{n_{k_{j}}}\right)_{j}$ of $\left(y_{n_{k}}\right)_{k}$ such that $y_{n_{k_{j}}} \rightarrow v$ (this, of course, is possible by boundedness of $\left.\left(y_{n}\right)_{n}\right)$. From the assumption $\left\|y_{n}-T y_{n}\right\| \rightarrow 0$ and demiclosedness of $T$ (see [1]) we have $v \in \operatorname{Fix}(T)$, and

$$
\begin{aligned}
\limsup _{n}\left\langle(I-f) \bar{p}, y_{n}-\bar{p}\right\rangle & =\lim _{j}\left\langle(I-f) \bar{p}, y_{n_{k}}-\bar{p}\right\rangle \\
& =\langle(I-f) \bar{p}, x-\bar{p}\rangle
\end{aligned}
$$

so the claim follows by (21).

(ii) It follows as in (i) since $S$ is demicloded too (see Theorem 4, (b)).

(iii) Select a subsequence $\left(y_{n_{k}}\right)_{k}$ of $\left(y_{n}\right)_{n}$ such that

$$
\limsup _{n}\left\langle(I-f) p_{0}, y_{n}-p_{0}\right\rangle=\lim _{k}\left\langle(I-f) p_{0}, y_{n_{k}}-p_{0}\right\rangle,
$$

where $p_{0}$ satisfies (25). Now select a subsequence $\left(y_{n_{k_{j}}}\right)_{j}$ of $\left(y_{n_{k}}\right)_{k}$ such that $y_{n_{k_{j}}} \rightarrow w$. Then, by demiclosedness of both $T$ and $S$, and by the hypotheses $\left\|y_{n}-T y_{n}\right\| \rightarrow 0$ and $\| y_{n}-$ $S y_{n} \| \rightarrow 0$, we obtain that $T w=S w=w$; that is, $w \in \operatorname{Fix}(T) \cap$ $\operatorname{Fix}(S)$. So the claim follows by $(25)$ and

$$
\begin{aligned}
& \limsup _{n}\left\langle(I-f) p_{0}, y_{n}-p_{0}\right\rangle \\
& \quad=\lim _{j}\left\langle(I-f) p_{0}, y_{n_{k}}-p_{0}\right\rangle=\left\langle(I-f) p_{0}, x-p_{0}\right\rangle .
\end{aligned}
$$


Lemma 9 (see [6]). Let $C$ be a nonempty closed convex subset of $H$ and let $T: C \rightarrow C$ be a nonexpansive mapping. Then $(I-T): C \rightarrow H$ is 1/2-inverse strongly monotone; that is,

$$
\frac{1}{2}\|(I-T) x-(I-T) y\|^{2} \leq\langle x-y,(I-T) x-(I-T) y\rangle .
$$

Lemma 10 (Maingé [25]). Let $\left(\tau_{n}\right)_{n}$ be real sequence that has a subsequence $\left(\tau_{n_{j}}\right)$ which satisfies $\tau_{n_{j}}<\tau_{n_{j}+1}$ for all $j$. Then the sequence of integers $(\delta(n))_{n}$ defined by $\delta(n)=\max \{k \leq n$ : $\left.\tau_{k}<\tau_{k+1}\right\}$ has the following properties:

(1) $\delta(n) \leq \delta(n+1)$;

(2) $\delta(n) \rightarrow \infty$ as $n \rightarrow \infty$;

(3) $\tau_{\delta(n)}<\tau_{\delta(n)+1}$;

(4) $\tau_{n}<\tau_{\delta(n)+1}$.

Theorem 11. Let

$$
\begin{aligned}
& x_{1} \in C, \\
& x_{n+1}=\alpha_{n} f\left(x_{n}\right) \\
& +\left(1-\alpha_{n}\right)\left[\beta_{n} T_{\omega} x_{n}+\left(1-\beta_{n}\right) S_{\omega} x_{n}\right], \quad n \geq 1
\end{aligned}
$$

with $\left(\alpha_{n}\right)_{n} \subset(0,1), \alpha_{n} \rightarrow 0$ and $\sum_{n} \alpha_{n}=\infty$. Then one has the following.

(i) If $\sum_{n}\left(1-\beta_{n}\right)<\infty$ and $\sum_{n}\left|\alpha_{n}-\alpha_{n+1}\right|<\infty$, then $\left(x_{n}\right)$ strongly converges to $\bar{p}=P_{\mathrm{Fix}(T)} f(\bar{p})$ that is the unique point in $\operatorname{Fix}(T)$ that satisfies the variational inequality

$$
\langle(I-f) \bar{p}, x-\bar{p}\rangle \geq 0, \quad \forall x \in \operatorname{Fix}(T) .
$$

(ii) If $\sum_{n} \beta_{n}<\infty$, then $\left(x_{n}\right)_{n}$ converges strongly to $\widetilde{p}=$ $P_{\mathrm{Fix}(S)} f(\tilde{p})$ that is the unique point in $\operatorname{Fix}(S)$ that satisfies the variational inequality

$$
\langle(I-f) \tilde{p}, x-\tilde{p}\rangle \geq 0, \quad \forall x \in \operatorname{Fix}(S) .
$$

(iii) If $\operatorname{lim~inf}_{n} \beta_{n}\left(1-\beta_{n}\right)>0$, then $\left(x_{n}\right)$ strongly converges to $p_{0}=P_{\mathrm{Fix}(T) \cap \mathrm{Fix}(S)} f\left(p_{0}\right)$ which is the unique point in $\operatorname{Fix}(T) \cap \operatorname{Fix}(S)$ that satisfies the variational inequality

$$
\left\langle(I-f) p_{0}, x-p_{0}\right\rangle \geq 0, \quad \forall x \in \operatorname{Fix}(T) \cap \operatorname{Fix}(S) .
$$

Proof. By Lemma 7, we obtain that $\left(x_{n}\right)_{n}$ is bounded.

Proof of (i). Let $\bar{p}$ be as in (i) of Lemma 8; that is,

$$
\langle(I-f) \bar{p}, x-\bar{p}\rangle \geq 0, \quad \forall x \in \operatorname{Fix}(T) .
$$

Step 1. One has $\lim \sup _{n}\left(\left\|x_{n}-\bar{p}\right\|-\left\|x_{n+1}-\bar{p}\right\|\right)=0$.

Proof of Step 1. This immediately follows by the asymptotic regularity of $\left(x_{n}\right)_{n}$. So we prove that $\left(x_{n}\right)$ is asymptotically regular; that is, $\left\|x_{n}-x_{n+1}\right\| \rightarrow 0$ :

$$
\begin{aligned}
x_{n+1}-x_{n}= & \alpha_{n} f\left(x_{n}\right)+\left(1-\alpha_{n}\right) U_{n} x_{n}-\alpha_{n-1} f\left(x_{n-1}\right) \\
& -\left(1-\alpha_{n-1}\right) U_{n-1} x_{n-1} \pm\left(1-\alpha_{n}\right) U_{n-1} x_{n-1} \\
= & \left(1-\alpha_{n}\right)\left[U_{n} x_{n}-U_{n-1} x_{n-1}\right] \\
& +\left(\alpha_{n-1}-\alpha_{n}\right) U_{n-1} x_{n-1} \\
& -\alpha_{n-1} f\left(x_{n-1}\right)+\alpha_{n} f\left(x_{n}\right)-\alpha_{n} f\left(x_{n-1}\right) \\
& +\alpha_{n} f\left(x_{n-1}\right) \\
= & \left(1-\alpha_{n}\right)\left[U_{n} x_{n}-U_{n-1} x_{n-1}\right] \\
& +\left(\alpha_{n-1}-\alpha_{n}\right)\left(U_{n-1} x_{n-1}-f\left(x_{n-1}\right)\right) \\
& +\alpha_{n}\left(f\left(x_{n}\right)-f\left(x_{n-1}\right)\right) \\
= & \left(\alpha_{n-1}-\alpha_{n}\right)\left(U_{n-1} x_{n-1}-f\left(x_{n-1}\right)\right) \\
& +\alpha_{n}\left(f\left(x_{n}\right)-f\left(x_{n-1}\right)\right) \\
& +\left(1-\alpha_{n}\right)\left[\beta_{n} T_{\omega} x_{n}+\left(1-\beta_{n}\right) S_{\omega} x_{n}\right. \\
& \quad-\beta_{n-1} T_{\omega} x_{n-1}-\left(1-\beta_{n-1}\right) S_{\omega} x_{n-1} \\
& \left.\quad \pm \beta_{n} T_{\omega} x_{n-1} \pm\left(1-\beta_{n}\right) S_{\omega} x_{n-1}\right] \\
& \left.+\left(\beta_{n}-\beta_{n-1}\right)\left(T_{\omega} x_{n-1}-S \omega x_{n-1}\right)\right] . \\
& +\alpha_{n-1}\left(f\left(\alpha_{n}\right)-f\left(x_{n-1}\right)\right)\left(U_{n-1} x_{n-1}-f\left(x_{n-1}\right)\right) \\
& +\left(\beta_{n}\left(T_{\omega} x_{n}-T_{\omega} x_{n-1}\right)\right. \\
& +\left(1-\beta_{n}\right)\left(S_{\omega} x_{n}-S_{\omega} x_{n-1}\right) \\
& \left.\quad+\alpha_{n}\right)
\end{aligned}
$$

So

$$
\begin{aligned}
\left\|x_{n+1}-x_{n}\right\| \leq & \left|\alpha_{n-1}-\alpha_{n}\right| O(1)+\alpha_{n} \rho\left\|x_{n}-x_{n-1}\right\| \\
& +\left(1-\alpha_{n}\right)\left[\beta_{n}\left\|x_{n}-x_{n-1}\right\|+\left(1-\beta_{n}\right) O(1)\right. \\
& \left.\quad+\left|\beta_{n}-\beta_{n-1}\right| O(1)\right] \\
= & \left(\left(1-\alpha_{n}\right) \beta_{n}+\alpha_{n} \rho\right)\left\|x_{n}-x_{n-1}\right\|+\gamma_{n} \\
= & 1-\left[1-\left(\left(1-\alpha_{n}\right) \beta_{n}+\alpha_{n} \rho\right)\right]\left\|x_{n}-x_{n-1}\right\|+\gamma_{n},
\end{aligned}
$$

where $\gamma_{n}=\left|\alpha_{n}-\alpha_{n-1}\right| O(1)+\left(1-\alpha_{n}\right)\left(1-\beta_{n}\right) O(1)+\mid \beta_{n}-$ $\beta_{n-1} \mid O(1)$ is such that $\sum_{n} \gamma_{n}<\infty$, thanks to the assumptions $\sum_{n}\left|\alpha_{n}-\alpha_{n-1}\right|<\infty$ and $\sum_{n}\left(1-\beta_{n}\right)<\infty$.

So if we put $\delta_{n}=1-\beta_{n}+\alpha_{n} \beta_{n}-\alpha_{n} \rho$ we have

$$
\left\|x_{n+1}-x_{n}\right\| \leq\left(1-\delta_{n}\right)\left\|x_{n-1}-x_{n}\right\|+\gamma_{n} .
$$


From the assumption $\sum_{n} \alpha_{n}=\infty$ we deduce immediately $\sum_{n} \delta_{n}=\infty$. This is sufficient for Xu's Lemma 6, to conclude that $\left(x_{n}\right)_{n}$ is asymptotically regular.

Step 2. One has $\left\|x_{n}-T_{\omega} x_{n}\right\| \rightarrow 0$, and $\left\|x_{n}-T x_{n}\right\|=$ $(1 / \omega)\left\|x_{n}-T_{\omega} x_{n}\right\| \rightarrow 0$.

Proof of Step 2. We define an auxiliary sequence $\left(z_{n}\right)_{n}$ by

$$
z_{n+1}=\alpha_{n} f\left(x_{n}\right)+\left(1-\alpha_{n}\right) T_{\omega} x_{n} .
$$

Observe that

$$
\left\|z_{n+1}-T_{\omega} x_{n}\right\|=\alpha_{n}\left\|f\left(x_{n}\right)-T_{\omega} x_{n}\right\| \longrightarrow 0
$$

and so

$$
\begin{aligned}
\left\|z_{n+1}-x_{n+1}\right\|= & \| \alpha_{n} f\left(x_{n}\right)+\left(1-\alpha_{n}\right) T_{\omega} x_{n} \\
& -\alpha_{n} f\left(x_{n}\right)-\left(1-\alpha_{n}\right) U_{n} x_{n} \| \\
= & \left(1-\alpha_{n}\right)\left\|T_{\omega} x_{n}-U x_{n}\right\| \\
= & \left(1-\alpha_{n}\right)\left\|T_{\omega} x_{n}-\beta_{n} T_{\omega} x_{n}-\left(1-\beta_{n}\right) S_{\omega} x_{n}\right\| \\
= & \left(1-\alpha_{n}\right)\left(1-\beta_{n}\right)\left\|T_{\omega} x_{n}-S_{\omega} x_{n}\right\| \longrightarrow 0 ;
\end{aligned}
$$

hence we get

$$
\sum_{n}\left\|x_{n}-z_{n}\right\|<\infty
$$

Now

$$
\begin{gathered}
\left\|z_{n+1}-\bar{p}\right\|^{2}=\| \alpha_{n} f\left(x_{n}\right)+\left(1-\alpha_{n}\right)(1-\omega) x_{n} \\
+\left(1-\alpha_{n}\right) \omega T x_{n}-\bar{p} \|^{2} \\
=\|\left[\left(1-\alpha_{n}\right) \omega\left(T x_{n}-x_{n}\right)+x_{n}-\bar{p}\right] \\
+\alpha_{n}\left(f\left(x_{n}\right)-x_{n}\right) \|^{2}
\end{gathered}
$$

(by the well known inequality

$$
\begin{aligned}
& \left.\|x+y\|^{2} \leq\|x\|^{2}+2\langle y, x+y\rangle\right) \\
\leq & \left\|\left(1-\alpha_{n}\right) \omega\left(T x_{n}-x_{n}\right)+x_{n}-\bar{p}\right\|^{2} \\
& +2 \alpha_{n}\left\langle f\left(x_{n}\right)-x_{n}, z_{n+1}-\bar{p}\right\rangle \\
\leq & \left(1-\alpha_{n}\right)^{2} \omega^{2}\left\|T x_{n}-x_{n}\right\|^{2}+\left\|x_{n}-\bar{p}\right\|^{2} \\
& -2\left(1-\alpha_{n}\right) \omega\left\langle x_{n}-\bar{p}, x_{n}-T x_{n}\right\rangle \\
& +2 \alpha_{n}\left\|f\left(x_{n}\right)-x_{n}\right\|\left\|z_{n+1}-\bar{p}\right\| \\
\leq & \left(1-\alpha_{n}\right)^{2} \omega^{2}\left\|T x_{n}-x_{n}\right\|^{2}+\left\|x_{n}-\bar{p}\right\|^{2}
\end{aligned}
$$

$$
\begin{aligned}
& -2\left(1-\alpha_{n}\right) \omega\left\langle x_{n}-\bar{p},(I-T) x_{n}-(I-T) \bar{p}\right\rangle \\
& +2 \alpha_{n}\left\|f\left(x_{n}\right)-x_{n}\right\|\left\|z_{n+1}-\bar{p}\right\|
\end{aligned}
$$

(by the inverse strong monotonicity

$$
\text { of }(I-T) \text {, Lemma 9) }
$$

$$
\begin{aligned}
\leq & \left(1-\alpha_{n}\right)^{2} \omega^{2}\left\|T x_{n}-x_{n}\right\|^{2}+\left\|x_{n}-\bar{p}\right\|^{2} \\
& -\left(1-\alpha_{n}\right) \omega\left\|(I-T) x_{n}-(I-T) \bar{p}\right\|^{2} \\
& +2 \alpha_{n}\left\|f\left(x_{n}\right)-x_{n}\right\|\left\|z_{n+1}-\bar{p}\right\| \\
= & \left\|x_{n}-\bar{p}\right\|^{2}-\left(1-\alpha_{n}\right) \omega\left[1-\left(1-\alpha_{n}\right) \omega\right] \\
& \times\left\|x_{n}-T x_{n}\right\|^{2}+2 \alpha_{n}\left\|f\left(x_{n}\right)-x_{n}\right\|\left\|z_{n+1}-\bar{p}\right\|
\end{aligned}
$$

and hence

$$
\begin{aligned}
0 \leq & \left(1-\alpha_{n}\right) \omega\left[1-\left(1-\alpha_{n}\right) \omega\right]\left\|x_{n}-T x_{n}\right\|^{2} \\
\leq & -\left\|z_{n+1}-\bar{p}\right\|^{2}+\left\|x_{n}-\bar{p}\right\|^{2} \\
& +2 \alpha_{n}\left\|f\left(x_{n}\right)-x_{n}\right\|\left\|z_{n+1}-\bar{p}\right\| .
\end{aligned}
$$

Passing to lim $\sup _{n}$, the last member goes to zero thanks to Step 1, to boundedness of $\left(x_{n}\right)_{n}$ and (41). So we obtain

$$
\omega(1-\omega) \lim _{n}\left\|x_{n}-T x_{n}\right\|=0 .
$$

From this immediately we have also $\left\|z_{n}-T z_{n}\right\| \rightarrow 0$.

From Step 2 and Lemma 8(i) we obtain

$$
\limsup _{n}\left\langle(I-f) \bar{p}, x_{n}-\bar{p}\right\rangle \geq 0
$$

Moreover, from Step 2 and Lemma 8 we also have

$$
\limsup _{n}\left\langle(I-f) \bar{p}, z_{n}-\bar{p}\right\rangle \geq 0
$$

Step 3. One has $z_{n} \rightarrow \bar{p}$.

Proof of Step 3. By using the auxiliary sequence $\left(z_{n}\right)_{n}$, we can write $x_{n}$ as

$$
x_{n+1}=z_{n+1}+\left(1-\beta_{n}\right) E_{n},
$$

where $E_{n}=\left(1-\alpha_{n}\right)\left(S_{\omega} x_{n}-T_{\omega} x_{n}\right)$ is a bounded sequence and so

$$
\left\|x_{n}-z_{n}\right\| \leq\left(1-\beta_{n}\right) O(1),
$$




$$
\begin{aligned}
\left\|z_{n+1}-\bar{p}\right\|^{2}= & \left\|\alpha_{n}\left(f\left(x_{n}\right)-\bar{p}\right)+\left(1-\alpha_{n}\right)\left(T_{\omega} x_{n}-\bar{p}\right)\right\|^{2} \\
= & \left(1-\alpha_{n}\right)^{2}\left\|T_{\omega} x_{n}-\bar{p}\right\|^{2}+\alpha_{n}^{2}\left\|f\left(x_{n}\right)-\bar{p}\right\|^{2} \\
& +2 \alpha_{n}\left(1-\alpha_{n}\right)\left\langle f\left(x_{n}\right)-\bar{p}, T_{\omega} x_{n}-\bar{p}\right\rangle \\
\leq & \left(1-2 \alpha_{n}+\alpha_{n}^{2}\right)\left\|x_{n}-\bar{p}\right\|^{2}+\alpha_{n}^{2}\left\|f\left(x_{n}\right)-\bar{p}\right\|^{2} \\
& +2 \alpha_{n}\left(1-\alpha_{n}\right)\left\langle f\left(x_{n}\right)-f(\bar{p}), T_{\omega} x_{n}-\bar{p}\right\rangle \\
& +2 \alpha_{n}\left(1-\alpha_{n}\right)\left\langle f(\bar{p})-\bar{p}, T_{\omega} x_{n}-\bar{p}\right\rangle \\
\leq & \left(1-2 \alpha_{n}+\alpha_{n}^{2}\right)\left\|x_{n}-\bar{p}\right\|^{2}+\alpha_{n}^{2}\left\|f\left(x_{n}\right)-\bar{p}\right\|^{2} \\
& +2 \alpha_{n}\left(1-\alpha_{n}\right) \rho\left\|x_{n}-\bar{p}\right\|^{2} \\
& +2 \alpha_{n}\left(1-\alpha_{n}\right)\left\langle f(\bar{p})-\bar{p}, T_{\omega} x_{n}-x_{n}\right\rangle \\
& +2 \alpha_{n}\left(1-\alpha_{n}\right)\left\langle f(\bar{p})-\bar{p}, x_{n}-\bar{p}\right\rangle \\
\leq & {\left[1-2 \alpha_{n}+\alpha_{n}^{2}+2 \alpha_{n}\left(1-\alpha_{n}\right) \rho\right] } \\
& \times\left(\left\|x_{n}-z_{n}\right\|+\left\|z_{n}-\bar{p}\right\|\right)^{2} \\
& +2 \alpha_{n}\left(1-\alpha_{n}\right)\left\langle f(\bar{p})-\bar{p}, T_{\omega} x_{n}-x_{n}\right\rangle \\
& +2 \alpha_{n}\left(1-\alpha_{n}\right)\left\langle f(\bar{p})-\bar{p}, x_{n}-\bar{p}\right\rangle \\
& +\alpha_{n}^{2}\left\|f\left(x_{n}\right)-\bar{p}\right\|^{2} \\
= & {\left[1-2 \alpha_{n}+\alpha_{n}^{2}+2 \alpha_{n}\left(1-\alpha_{n}\right) \rho\right]\left\|z_{n}-\bar{p}\right\|^{2} } \\
& +\left(1-\beta_{n}\right) O(1) \\
& +2 \alpha_{n}\left(1-\alpha_{n}\right)\left\langle f(\bar{p})-\bar{p}, T_{\omega} x_{n}-x_{n}\right\rangle \\
& \times 2 \alpha_{n}\left(1-\alpha_{n}\right)\left\langle f(\bar{p})-\bar{p}, x_{n}-\bar{p}\right\rangle \\
& +\alpha_{n}^{2}\left\|f\left(x_{n}\right)-\bar{p}\right\|^{2} . \\
& \\
&
\end{aligned}
$$

So putting $\delta_{n}=\alpha_{n}\left[2-\alpha_{n}-2 \rho\left(1-\alpha_{n}\right)\right], \gamma_{n}=\left(1-\beta_{n}\right) O(1)$, and

$$
\begin{aligned}
\sigma_{n}= & 2\left(1-\alpha_{n}\right)\left\langle f(\bar{p})-\bar{p}, T_{\omega} x_{n}-x_{n}\right\rangle \\
& +2\left(1-\alpha_{n}\right)\left\langle f(\bar{p})-\bar{p}, x_{n}-\bar{p}\right\rangle+\alpha_{n}\left\|f\left(x_{n}\right)-\bar{p}\right\|^{2}
\end{aligned}
$$

one has easily that $\delta_{n} \in(0,1), \sum_{n} \delta_{n}=\infty, \sum_{n} \gamma_{n}<\infty$, and

$$
\limsup _{n} \sigma_{n}=\limsup _{n}\left\langle x_{n}-\bar{p}, f(\bar{p})-\bar{p}\right\rangle \leq 0 .
$$

Thus, we can rewrite (50) as

$$
\left\|z_{n+1}-\bar{p}\right\|^{2} \leq\left(1-\delta_{n}\right)\left\|z_{n}-\bar{p}\right\|^{2}+\alpha_{n} \sigma_{n}+\gamma_{n} .
$$

This is sufficient, for Xu's Lemma 6 , to conclude that $z_{n} \rightarrow \bar{p}$. Lastly, by (49) immediately follows $x_{n} \rightarrow \bar{p}$.
Proof of (ii). Rewrite $x_{n+1}$ as

$$
x_{n+1}=\alpha_{n} f\left(x_{n}\right)+\left(1-\alpha_{n}\right) S_{\omega} x_{n}+\beta_{n} E_{n},
$$

where $E_{n}=\left(1-\alpha_{n}\right)\left(T_{\omega} x_{n}+S_{\omega} x_{n}\right)$ is bounded (i.e., $\left\|E_{n}\right\| \leq$ $O(1))$.

Now,

$$
\begin{aligned}
\left\|x_{n+1}-\widetilde{p}\right\|^{2}= & \| \alpha_{n} f\left(x_{n}\right)+\left(1-\alpha_{n}\right)(1-\omega) x_{n} \\
& +\left(1-\alpha_{n}\right) \omega S x_{n}+\beta E_{n}-\tilde{p} \|^{2} \\
= & \|\left[\left(1-\alpha_{n}\right) \omega\left(S x_{n}-x_{n}\right)+x_{n}-\tilde{p}\right] \\
& +\left[\alpha_{n}\left(f\left(x_{n}\right)-x_{n}\right)+\beta_{n} E_{n}\right] \|^{2}
\end{aligned}
$$

(by the well known inequality

$$
\begin{aligned}
& \left.\|x+y\|^{2} \leq\|x\|^{2}+2\langle y, x+y\rangle\right) \\
\leq & \left\|\left(1-\alpha_{n}\right) \omega\left(S x_{n}-x_{n}\right)+x_{n}-\tilde{p}\right\|^{2} \\
& +2 \alpha_{n}\left\langle f\left(x_{n}\right)-x_{n}, x_{n+1}-\tilde{p}\right\rangle+\beta_{n} O(1) \\
\leq & \left(1-\alpha_{n}\right)^{2} \omega^{2}\left\|S x_{n}-x_{n}\right\|^{2}+\left\|x_{n}-\tilde{p}\right\|^{2} \\
& -2\left(1-\alpha_{n}\right) \omega\left\langle x_{n}-S x_{n}, x_{n}-\tilde{p}\right\rangle \\
& +2 \alpha_{n}\left\|f\left(x_{n}\right)-x_{n}\right\|\left\|x_{n+1}-\tilde{p}\right\|+\beta_{n} O(1)
\end{aligned}
$$

(by (c) of Theorem 4)

$$
\begin{aligned}
\leq & \left(1-\alpha_{n}\right)^{2} \omega^{2}\left\|S x_{n}-x_{n}\right\|^{2}+\left\|x_{n}-\tilde{p}\right\|^{2} \\
& -2\left(1-\alpha_{n}\right) \omega\left[\left\|x_{n}-S x_{n}\right\|^{2}-\frac{1}{2}\left\|x_{n}-S x_{n}\right\|^{2}\right] \\
& +2 \alpha_{n}\left\|f\left(x_{n}\right)-x_{n}\right\|\left\|x_{n+1}-\tilde{p}\right\|+\beta_{n} O(1) \\
= & \left\|x_{n}-\tilde{p}\right\|^{2} \\
& +\left[\left(1-\alpha_{n}\right)^{2} \omega^{2}-\left(1-\alpha_{n}\right) \omega\right]\left\|x_{n}-S x_{n}\right\|^{2} \\
& +2 \alpha_{n}\left\|f\left(x_{n}\right)-x_{n}\right\|\left\|x_{n+1}-\tilde{p}\right\|+\beta_{n} O(1) \\
= & \left\|x_{n}-\tilde{p}\right\|^{2} \\
& -\left(1-\alpha_{n}\right) \omega\left[1-\left(1-\alpha_{n}\right) \omega\right]\left\|x_{n}-S x_{n}\right\|^{2} \\
& +2 \alpha_{n}\left\|f\left(x_{n}\right)-x_{n}\right\|\left\|x_{n+1}-\tilde{p}\right\|+\beta_{n} O(1)
\end{aligned}
$$

and hence

$$
\begin{aligned}
& \left(1-\alpha_{n}\right) \omega\left[1-\left(1-\alpha_{n}\right) \omega\right]\left\|x_{n}-S x_{n}\right\|^{2} \\
& -2 \alpha_{n}\left\|f\left(x_{n}\right)-x_{n}\right\|\left\|x_{n+1}-\tilde{p}\right\| \\
& -\beta_{n} O(1) \leq\left\|x_{n}-\tilde{p}\right\|^{2}-\left\|x_{n+1}-\tilde{p}\right\|^{2} .
\end{aligned}
$$

Now we distinguish two alternatives. 
Alternative 1. $\left(\left\|x_{n}-\widetilde{p}\right\|\right)_{n}$ is definitively nonincreasing.

Then there exists $\lim _{n}\left\|x_{n}-\tilde{p}\right\|^{2}$ and so, passing to the lim sup in (56), we obtain

$$
\left\|x_{n}-S x_{n}\right\| \longrightarrow 0, \quad\left\|x_{n}-S_{\omega} x_{n}\right\|=\omega\left\|x_{n}-S x_{n}\right\| \longrightarrow 0 .
$$

By Lemma 8(ii) it follows that

$$
\langle(I-f) \tilde{p}, x-\tilde{p}\rangle \geq 0 .
$$

So

$$
\begin{aligned}
\left\|x_{n+1}-\widetilde{p}\right\|^{2}= & \| \alpha_{n}\left(f\left(x_{n}\right)-\widetilde{p}\right) \\
& +\left(1-\alpha_{n}\right)\left(S_{\omega} x_{n}-\tilde{p}\right)+\beta_{n} E_{n} \|^{2} \\
\leq & \left\|\alpha_{n}\left(f\left(x_{n}\right)-\widetilde{p}\right)+\left(1-\alpha_{n}\right)\left(S_{\omega} x_{n}-\widetilde{p}\right)\right\|^{2} \\
& +\beta_{n} O(1) \\
= & \left(1-\alpha_{n}\right)^{2}\left\|S_{\omega} x_{n}-\widetilde{p}\right\|^{2}+\alpha_{n}^{2} O(1) \\
& +2 \alpha_{n}\left(1-\alpha_{n}\right)\left\langle f\left(x_{n}\right)-\widetilde{p}, S_{\omega} x_{n}-\widetilde{p}\right\rangle \\
& +\beta_{n} O(1)
\end{aligned}
$$

(by the quasi-nonexpansivity of $S_{\omega}$ )$$
\leq\left(1-2 \alpha_{n}+\alpha_{n}^{2}\right)\left\|x_{n}-\tilde{p}\right\|^{2}+\alpha_{n}^{2} O(1)
$$$$
+\beta_{n} O(1)
$$$$
+2 \alpha_{n}\left(1-\alpha_{n}\right)\left\langle f\left(x_{n}\right)-f(\tilde{p}), S_{\omega} x_{n}-\tilde{p}\right\rangle
$$$$
+2 \alpha_{n}\left(1-\alpha_{n}\right)\left\langle f(\tilde{p})-\tilde{p}, S_{\omega} x_{n}-\tilde{p}\right\rangle
$$$$
\leq\left(1-2 \alpha_{n}+\alpha_{n}^{2}+2 \alpha_{n}\left(1-\alpha_{n}\right) \rho\right)\left\|x_{n}-\tilde{p}\right\|^{2}
$$$$
+\alpha_{n}^{2} O(1)+\beta_{n} O(1)
$$$$
+2 \alpha_{n}\left(1-\alpha_{n}\right)\left\langle f(\tilde{p})-\tilde{p}, S_{\omega} x_{n}-x_{n}\right\rangle
$$$$
+2 \alpha_{n}\left(1-\alpha_{n}\right)\left\langle f(\tilde{p})-\tilde{p}, x_{n}-\tilde{p}\right\rangle
$$$$
=\left(1-2 \alpha_{n}+\alpha_{n}^{2}+2 \alpha_{n}\left(1-\alpha_{n}\right) \rho\right)\left\|x_{n}-\widetilde{p}\right\|^{2}
$$$$
+\alpha_{n}^{2} O(1)+\alpha_{n}\left[\left\|S_{\omega} x_{n}-x_{n}\right\| O(1)\right.
$$$$
\left.+(1-\alpha)\left\langle f(\widetilde{p})-\widetilde{p}, x_{n}-\tilde{p}\right\rangle\right]
$$$$
+\beta_{n} O(1) .
$$

So, as in Step 1, thanks to (57), (58), and Xu's Lemma, we obtain $x_{n} \rightarrow \tilde{p}$.

Alternative 2. $\left(\left\|x_{n}-\widetilde{p}\right\|\right)_{n}$ is not definitively nonincreasing. that

This means that there exists a subsequence $\left(x_{n_{k}}\right)_{k}$ such

$$
\left\|x_{n_{k}}-\tilde{p}\right\|^{2} \leq\left\|x_{n_{k}+1}-\tilde{p}\right\|^{2} .
$$

Then, thanks to Maingé's Lemma, we know that there exists a sequence of integers $(\delta(n))_{n}$ that satisfies the following.

(i) $\delta(n)$ is nondecreasing, (ii) $\delta(n) \rightarrow \infty$, (iii) $\left\|x_{\delta(n)}-\widetilde{p}\right\|^{2}<\left\|x_{\delta(n)+1}-\widetilde{p}\right\|^{2}$, and (iv) $\left\|x_{n}-\widetilde{p}\right\|<\left\|x_{\delta(n)+1}-\widetilde{p}\right\|$, for all $n \geq n_{1}$.

Consequently,

$$
\begin{aligned}
0 & \leq \liminf _{n}\left\|x_{\delta(n)+1}-\tilde{p}\right\|-\left\|x_{\delta(n)}-\tilde{p}\right\| \\
& \leq \limsup _{n}\left\|x_{\delta(n)+1}-\tilde{p}\right\|-\left\|x_{\delta(n)}-\tilde{p}\right\| \\
& \leq \limsup _{n}\left\|x_{n+1}-\tilde{p}\right\|-\left\|x_{n}-\tilde{p}\right\| \\
& \leq \limsup _{n}\left\|\alpha_{n}\left(f\left(x_{n}\right)-S_{\omega} x_{n}\right)+\left(S_{\omega} x_{n}-\tilde{p}\right)+\beta_{n} E_{n}\right\| \\
& \quad-\left\|x_{n}-\tilde{p}\right\|
\end{aligned}
$$

(by the quasi-nonexpansivity of $S_{\omega}$ )

$$
\begin{gathered}
\leq \lim \sup \alpha_{n} O(1)+\left\|x_{n}-\tilde{p}\right\| \\
\quad+\beta_{n} O(1)-\left\|x_{n}-\tilde{p}\right\|=0 .
\end{gathered}
$$

So

$$
\lim _{n}\left(\left\|x_{\delta(n)+1}-\tilde{p}\right\|-\left\|x_{n}-\widetilde{p}\right\|\right)=0
$$

If we rewrite (56) as

$$
\begin{aligned}
&\left(1-\alpha_{n}\right) \omega\left[1-\left(1-\alpha_{n}\right) \omega\right]\left\|x_{n}-S x_{n}\right\|^{2} \\
& \leq \alpha_{n} O(1)+\beta_{n} O(1)+\left\|x_{n}-\widetilde{p}\right\|^{2}-\left\|x_{n+1}-\widetilde{p}\right\|^{2} \\
& \leq \alpha_{n} O(1)+\beta_{n} O(1) \\
& \quad+\left(\left\|x_{n}-\tilde{p}\right\|-\left\|x_{n+1}-\widetilde{p}\right\|\right) O(1),
\end{aligned}
$$

then (62) implies that

$$
\left\|x_{\delta(n)}-S x_{\delta(n)}\right\| \longrightarrow 0
$$

and this, in turn, by using Lemma 8(ii), means that

$$
\lim \sup \left\langle(I-f) \tilde{p}, y_{n}-\tilde{p}\right\rangle \geq 0 .
$$

At this point it is clear that we can continue as in Alternative 1 and we obtain $\left\|x_{\delta(n)}-\tilde{p}\right\| \rightarrow 0$.

Then (62) furnishes

$$
\left\|x_{\delta(n)+1}-\tilde{p}\right\| \longrightarrow 0
$$

and finally by property (iv) of Maingés Lemma, that is, $\| x_{n}-$ $\tilde{p}\|<\| x_{\delta(n)+1}-\tilde{p} \|$, for all $n \geq n_{1}$, we point out that $x_{n} \rightarrow \widetilde{p}$.

Proof of (iii). Let be $p_{0}$ as in (iii) of Lemma 8; that is,

$$
\left\langle(I-f) p_{0}, x-p_{0}\right\rangle \geq 0, \quad \forall x \in \operatorname{Fix}(T) \cap \operatorname{Fix}(S) .
$$


Now,

$$
\begin{aligned}
\left\|U_{n} x_{n}-p_{0}\right\|^{2}= & \left\|\beta_{n}\left(T \omega x_{n}-p_{0}\right)+\left(1-\beta_{n}\right)\left(S_{\omega} x_{n}-p_{0}\right)\right\|^{2} \\
= & \beta_{n}\left\|T \omega x_{n}-p_{0}\right\|^{2}+\left(1-\beta_{n}\right)\left\|S_{\omega} x_{n}-p_{0}\right\|^{2} \\
& -\beta_{n}\left(1-\beta_{n}\right)\left\|T_{\omega} x_{n}-S_{\omega} x_{n}\right\|^{2} \\
& \quad \text { by the quasi-nonexpansivity of } T_{\omega}, \\
& \quad \text { by quasi firmly nonexpansivity } \\
& \text { of } \left.S_{\omega} \text { and Theorem } 4(\mathrm{i})\right) \\
\leq & \beta_{n}\left\|x_{n}-p_{0}\right\|^{2}+\left(1-\beta_{n}\right)\left\|x_{n}-p_{0}\right\|^{2} \\
& -\left(1-\beta_{n}\right)(1-\omega)\left\|x_{n}-S_{\omega} x_{n}\right\|^{2} \\
& -\beta_{n}\left(1-\beta_{n}\right)\left\|T_{\omega} x_{n}-S_{\omega} x_{n}\right\|^{2} \\
&
\end{aligned}
$$

so

$$
\begin{aligned}
\left\|x_{n+1}-p_{0}\right\|^{2}= & \| \alpha_{n}\left(f\left(x_{n}\right)-p_{0}\right) \\
& +\left(1-\alpha_{n}\right)\left(U_{n} x_{n}-p_{0}\right) \|^{2} \\
\leq & \left\|U_{n} x_{n}-p_{0}\right\|^{2}+\alpha_{n}\left(\alpha_{n} O(1)+O(1)\right) \\
\leq & \text { by }(68) \\
\leq & \left\|x_{n}-p_{0}\right\|^{2}-\left(1-\beta_{n}\right)(1-\omega)\left\|x_{n}-S_{\omega} x_{n}\right\|^{2} \\
& \quad-\beta_{n}\left(1-\beta_{n}\right)\left\|T_{\omega} x_{n}-S_{\omega} x_{n}\right\|^{2} .
\end{aligned}
$$

From this we derive the following inequalities:

$$
\begin{aligned}
& \left(1-\beta_{n}\right)(1-\omega)\left\|x_{n}-S_{\omega} x_{n}\right\|^{2} \\
& \quad \leq\left\|x_{n}-p_{0}\right\|^{2}-\left\|x_{n+1}-p_{0}\right\|+\alpha_{n} O(1), \\
& \beta_{n}\left(1-\beta_{n}\right)\left\|T_{\omega} x_{n}-S_{\omega} x_{n}\right\|^{2} \\
& \quad \leq\left\|x_{n}-p_{0}\right\|^{2}-\left\|x_{n+1}-p_{0}\right\|^{2}+\alpha_{n} O(1) .
\end{aligned}
$$

Now, also here consider two alternatives.

Alternative 1. $\left(\left\|x_{n}-p_{0}\right\|\right)_{n}$ is definitively nonincreasing.

Then there exist $\lim \left\|x_{n}-p_{0}\right\|$ and $\left(\mid\left\|x_{n+1}-p_{0}\right\|-\| x_{n}-\right.$ $\left.p_{0} \| l\right) \rightarrow 0$.

So, passing to the lim sup in (71), the assumption $\lim \inf _{n} \beta_{n}\left(1-\beta_{n}\right)>0$ yields:

$$
\begin{gathered}
\left\|x_{n}-S_{\omega} x_{n}\right\| \longrightarrow 0, \\
\left\|S_{\omega} x_{n}-T_{\omega} x_{n}\right\| \longrightarrow 0 .
\end{gathered}
$$

Moreover, $x_{n}-S_{\omega} x_{n}=\omega\left(x_{n}-S x_{n}\right)$ so,

$$
\left\|x_{n}-S x_{n}\right\| \longrightarrow 0
$$

and $T_{\omega} x_{n}-S_{\omega} x_{n}=\omega\left(T x_{n}-S x_{n}\right)$ so

$$
\left\|T x_{n}-S x_{n}\right\| \longrightarrow 0
$$

so that from $x_{n}-T x_{n}=x_{n}-S x_{n}+S x_{n}-T x_{n}$ it follows at once that

$$
\left\|x_{n}-T x_{n}\right\| \longrightarrow 0
$$

From Lemma 8(iii) we obtain

$$
\lim \sup \left\langle(I-f) p_{0}, x_{n}-p_{0}\right\rangle \geq 0 .
$$

Further, from $U_{n} x_{n}-x_{n}=\beta_{n}\left(T_{\omega} x_{n}-x_{n}\right)+\left(1-\beta_{n}\right)\left(S_{\omega} x_{n}-x_{n}\right)$, we get

$$
\left\|U_{n} x_{n}-x_{n}\right\| \longrightarrow 0
$$

Now we are able to show that $x_{n} \rightarrow p_{0}$. Indeed,

$$
\begin{aligned}
\left\|x_{n+1}-p_{0}\right\|^{2}= & \|\left(1-\alpha_{n}\right)\left(U_{n} x_{n}-p_{0}\right) \\
& +\alpha_{n}\left(f\left(x_{n}\right)-p_{0}\right) \|^{2} \\
= & \left(1-\alpha_{n}\right)^{2}\left\|U_{n} x_{n}-p_{0}\right\|^{2} \\
& +\alpha_{n}^{2}\left\|f\left(x_{n}\right)-p_{0}\right\|^{2} \\
& +2\left(1-\alpha_{n}\right) \alpha_{n}\left\langle U_{n} x_{n}-p_{0}, f\left(x_{n}\right)-p_{0}\right\rangle \\
\leq & \left(1-2 \alpha_{n}+\alpha_{n}^{2}\right)\left\|x_{n}-p_{0}\right\|^{2} \\
& +\alpha_{n}^{2}\left\|f\left(x_{n}\right)-p_{0}\right\|^{2} \\
& +2\left(1-\alpha_{n}\right) \alpha_{n}\left\langle U_{n} x_{n}-p_{0}, f\left(x_{n}\right)-f\left(p_{0}\right)\right\rangle \\
& +2\left(1-\alpha_{n}\right) \alpha_{n}\left\langle U_{n} x_{n}-p_{0}, f\left(p_{0}\right)-p_{0}\right\rangle \\
\leq & {\left[1-2 \alpha_{n}+\alpha_{n}^{2}+2 \alpha_{n}\left(1-\alpha_{n}\right) \rho\right]\left\|x_{n}-p_{0}\right\|^{2} } \\
& +\alpha_{n}^{2}\left\|f\left(x_{n}\right)-p_{0}\right\|^{2} \\
& +2\left(1-\alpha_{n}\right) \alpha_{n}\left\langle U_{n} x_{n}-x_{n}, f\left(p_{0}\right)-p_{0}\right\rangle \\
& +2\left(1-\alpha_{n}\right) \alpha_{n}\left\langle x_{n}-p_{0}, f\left(p_{0}\right)-p_{0}\right\rangle . \\
& 2(78)
\end{aligned}
$$

So, put $\delta_{n}=\alpha_{n}\left[2-\alpha_{n}-2 \rho\left(1-\alpha_{n}\right)\right], \sigma_{n}=\alpha_{n}\left\|f\left(x_{n}\right)-p_{0}\right\|^{2}$ $+2\left(1-\alpha_{n}\right)\left\langle U_{n} x_{n}-x_{n}, f\left(p_{0}\right)-p_{0}\right\rangle+2\left(1-\alpha_{n}\right)\left\langle x_{n}-p_{0}, f\left(p_{0}\right)-\right.$ $\left.p_{0}\right\rangle$ one easily has $\delta_{n} \in(0,1), \sum_{n} \delta_{n}=\infty$, $\lim \sup _{n} \sigma_{n}=$ $\lim \sup _{n}\left\langle x_{n}-p_{0}, f\left(p_{0}\right)-p_{0}\right\rangle \leq 0$, and

$$
\left\|x_{n+1}-p_{0}\right\|^{2} \leq\left(1-\delta_{n}\right)\left\|x_{n}-p_{0}\right\|^{2}+\alpha_{n} \sigma_{n} .
$$

This is sufficient, for Xu's Lemma 6, to ensure that $x_{n} \rightarrow p_{0}$. 
Alternative 2. $\left(\left\|x_{n}-p_{0}\right\|\right)_{n}$ is not definitively nonincreasing.

This means that there exists a subsequence $\left(\left\|x_{n_{k}}-p_{0}\right\|\right)_{k}$ such that

$$
\left\|x_{n_{k}}-p_{0}\right\| \leq\left\|x_{n_{k}+1}-p_{0}\right\| .
$$

Then, thanks to Maingé's Lemma, we know that there exists a sequence of integers $\left(\delta_{n}\right)$ that satisfies the following.

(i) $\delta(n)$ is nondecreasing, (ii) $\delta(n) \rightarrow \infty$, (iii) $\left\|x_{\delta(n)}-p_{0}\right\|^{2}<\left\|x_{\delta(n)+1}-p_{0}\right\|^{2}$, and (iv) $\left\|x_{n}-p_{0}\right\|<$ $\left\|x_{\delta(n)+1}-p_{0}\right\|^{2}$, for all $n \geq n_{1}$.

Consequently,

$$
\begin{aligned}
0 & \leq \liminf _{n}\left\|x_{\delta(n)+1}-p_{0}\right\|-\left\|x_{\delta(n)}-p_{0}\right\| \\
& \leq \limsup _{n}\left\|x_{\delta(n)+1}-p_{0}\right\|-\left\|x_{\delta(n)}-p_{0}\right\| \\
& \leq \limsup _{n}\left\|x_{n+1}-\tilde{p}\right\|-\left\|x_{n}-\tilde{p}\right\| \\
& \leq \limsup _{n}\left[\left\|\alpha_{n}\left(f\left(x_{n}\right)-p_{0}\right)+\left(1-\alpha_{n}\right)\left(U_{n} x_{n}-p_{0}\right)\right\|\right. \\
& \left.\quad-\left\|x_{n}-p_{0}\right\|\right] \\
& \leq \lim \sup _{n}\left[\left\|f\left(x_{n}\right)-p_{0}\right\|-\left\|x_{n}-p_{0}\right\|\right]=0 .
\end{aligned}
$$

Hence

$$
\lim _{n}\left(\left\|x_{\delta(n)+1}-p_{0}\right\|-\left\|x_{\delta(n)}-p_{0}\right\|\right)=0
$$

So, passing to limsup on $\delta(n)$ in (71), one obtains, as in the Alternative 1,

$$
\begin{gathered}
\left\|x_{\delta(n)}-S_{\omega} x_{\delta(n)}\right\| \longrightarrow 0, \\
\left\|T_{\omega} x_{\delta(n)}-S_{\omega} x_{\delta(n)}\right\| \longrightarrow 0, \\
\left\|x_{\delta(n)}-S x_{\delta(n)}\right\| \longrightarrow 0, \\
\left\|T x_{\delta(n)}-S x_{\delta(n)}\right\| \longrightarrow 0, \\
\left\|x_{\delta(n)}-T x_{\delta(n)}\right\| \longrightarrow 0, \\
\left\|U_{\delta(n)} x_{\delta(n)}-x_{\delta(n)}\right\| \longrightarrow 0
\end{gathered}
$$

Again from Lemma 8(iii) it follows that

$$
\lim \sup \left\langle(I-f) p_{0}, x_{\delta(n)}-p_{0}\right\rangle \geq 0 .
$$

Following the reasoning of Alternative 1, one obtains that

$$
\left\|x_{\delta(n)}-p_{0}\right\| \longrightarrow 0 \text {. }
$$

Then (82) furnishes $\left\|x_{\delta(n)+1}-p_{0}\right\| \rightarrow 0$ and finally by the property (iv) of Maingé Lemma; that is,

$$
\left\|x_{n}-p_{0}\right\|<\left\|x_{\delta(n)+1}-p_{0}\right\|
$$

we obtain $x_{n} \rightarrow p_{0}$ as required.

Remark 12. The main result of this paper contains as a particular case the positive answer to the question raised by Kurokawa and Takahashi page 1567 in [26].
Remark 13. Our reasoning, different from that of Tian and Jin [27] and Deng et al. [28], has allowed us to prove our results without having $\omega<1 / 2$.

\section{Conflict of Interests}

The authors declare that there is no conflict of interests regarding the publication of this paper.

\section{Acknowledgment}

This project was funded by the Deanship of Scientific Research (DSR), King Abdulaziz University under Grant no. 29-130-35-HiCi. The authors, therefore, acknowledge technical and financial support of KAU.

\section{References}

[1] F. E. Browder and W. V. Petryshyn, "Construction of fixed points of nonlinear mappings in Hilbert space," Journal of Mathematical Analysis and Applications, vol. 20, no. 2, pp. 197228,1967

[2] Y. Song and X. Chai, "Halpern iteration for firmly type nonexpansive mappings," Nonlinear Analysis: Theory, Methods and Applications, vol. 71, no. 10, pp. 4500-4506, 2009.

[3] R. E. Bruck and S. Reich, "Nonexpansive projections and resolvents of accretive operators in Banach spaces," Houston Journal of Mathematics, vol. 3, no. 4, pp. 459-470, 1977.

[4] F. Kohsaka and W. Takahashi, "Fixed point theorems for a class of nonlinear mappings related to maximal monotone operators in Banach spaces," Archiv der Mathematik, vol. 91, no. 2, pp. 166$177,2008$.

[5] M. O. Osilike and F. O. Isiogugu, "Weak and strong convergence theorems for nonspreading-type mappings in Hilbert spaces," Nonlinear Analysis: Theory, Methods \& Applications, vol. 74, no. 5, pp. 1814-1822, 2011.

[6] K. Goebel and W. A. Kirk, Topics in Metric Fixed Point Theory, vol. 28 of Cambridge Studies in Advanced Mathematics, Cambridge University Press, Cambridge, UK, 1990.

[7] C. Byrne, "A unified treatment of some iterative algorithms in signal processing and image reconstruction," Inverse Problems, vol. 20, no. 1, pp. 103-120, 2004.

[8] H. Schaefer, "Uber die Methode sukzessiver Approximationen," Jahresbericht der Deutschen Mathematiker-Vereinigung, vol. 59, pp. 131-140, 1957.

[9] S. Iemoto and W. Takahashi, "Approximating common fixed points of nonexpansive mappings and nonspreading mappings in a Hilbert space," Nonlinear Analysis: Theory, Methods and Applications, vol. 71, no. 12, pp. 2082-2089, 2009.

[10] S. Saejung, "Halpern's iteration in Banach spaces," Nonlinear Analysis: Theory, Methods \& Applications, vol. 73, no. 10, pp. 3431-3439, 2010.

[11] A. Moudafi, "Krasnoselski-Mann iteration for hierarchical fixed-point problems," Inverse Problems, vol. 23, no. 4, pp. 16351640, 2007.

[12] F. Cianciaruso, G. Marino, A. Rugiano, and B. Scardamaglia, "On strong convergence of Halpern's iterative method using averaged type mappings," Journal of Applied Mathematics, vol. 2014, Article ID 473243, 10 pages, 2014. 
[13] A. Moudafi, "Viscosity approximation methods for fixed-points problems," Journal of Mathematical Analysis and Applications, vol. 241, no. 1, pp. 46-55, 2000.

[14] T. Suzuki, "Moudafi's viscosity approximations with MeirKeeler contractions," Journal of Mathematical Analysis and Applications, vol. 325, no. 1, pp. 342-352, 2007.

[15] G. Marino and H. K. Xu, "A general iterative method for nonexpansive mappings in Hilbert spaces," Journal of Mathematical Analysis and Applications, vol. 318, no. 1, pp. 43-52, 2006.

[16] H. K. Xu, "Viscosity approximation methods for nonexpansive mappings," Journal of Mathematical Analysis and Applications, vol. 298, no. 1, pp. 279-291, 2004.

[17] A. R. Khan, V. Kumar, and N. Hussain, "Analytical and numerical treatment of Jungck-type iterative schemes," Applied Mathematics and Computation, vol. 231, pp. 521-535, 2014.

[18] N. Hussain and W. Takahashi, "Weak and strong convergence theorems for semigroups of mappings without continuity in Hilbert spaces," Journal of Nonlinear and Convex Analysis, vol. 14, no. 4, pp. 769-783, 2013.

[19] N. Hussain, A. Rafiq, and L. B. Ciric, "Stability of the Ishikawa iteration scheme with errors for two strictly hemicontractive operators in Banach spaces," Fixed Point Theory and Applications, vol. 2012, article 160, 2012.

[20] L. C. Ceng, N. Husain, A. Latif, and J. C. Yao, "Strong convergence for solving general system of variational inequalities and fixed point problems in Banach spaces," Journal of Inequalities and Applications, vol. 2013, article 334, 2013.

[21] F. Cianciaruso, G. Marino, and L. Muglia, "Ishikawa iterations for equilibrium and fixed point problems for nonexpansive mappings in Hilbert spaces," Fixed Point Theory, vol. 9, no. 2, pp. 449-464, 2008.

[22] V. Colao, G. Marino, and H. Xu, "An iterative method for finding common solutions of equilibrium and fixed point problems," Journal of Mathematical Analysis and Applications, vol. 344, no. 1, pp. 340-352, 2008.

[23] F. Cianciaruso, G. Marino, and L. Muglia, "Iterative methods for equilibrium and fixed point problems for nonexpansive semigroups in Hilbert spaces," Journal of Optimization Theory and Applications, vol. 146, no. 2, pp. 491-509, 2010.

[24] H. K. Xu, "Iterative algorithms for nonlinear operators," Journal of the London Mathematical Society, vol. 66, no. 1, pp. 240-256, 2002.

[25] P. Maingé, "Strong convergence of projected subgradient methods for nonsmooth and nonstrictly convex minimization," SetValued Analysis, vol. 16, no. 7-8, pp. 899-912, 2008.

[26] Y. Kurokawa and W. Takahashi, "Weak and strong convergence theorems for nonspreading mappings in Hilbert spaces," Nonlinear Analysis: Theory, Methods \& Applications, vol. 73, no. 6, pp. 1562-1568, 2010.

[27] M. Tian and X. Jin, "A general iterative method for quasinonexpansive mappings in Hilbert space," Journal of Inequalities and Applications, vol. 2012, article 38, 2012.

[28] B. Deng, T. Chen, and Z. Li, "Cyclic iterative method for strictly pseudononspreading in hilbert space," Journal of Applied Mathematics, vol. 2012, Article ID 435676, 15 pages, 2012. 


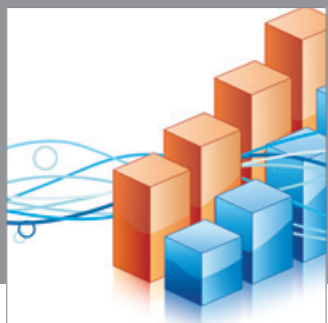

Advances in

Operations Research

mansans

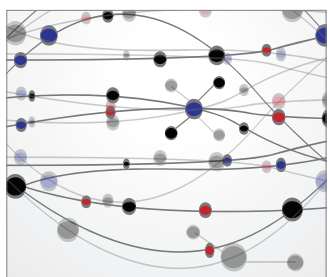

The Scientific World Journal
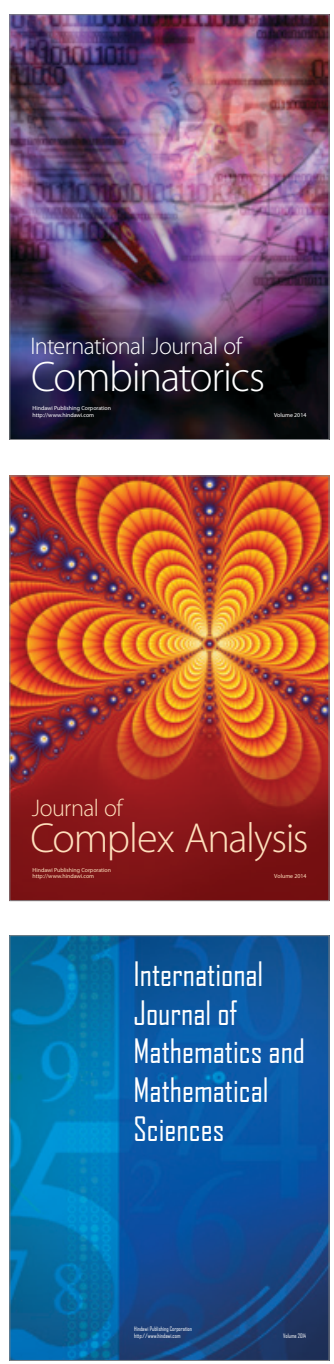
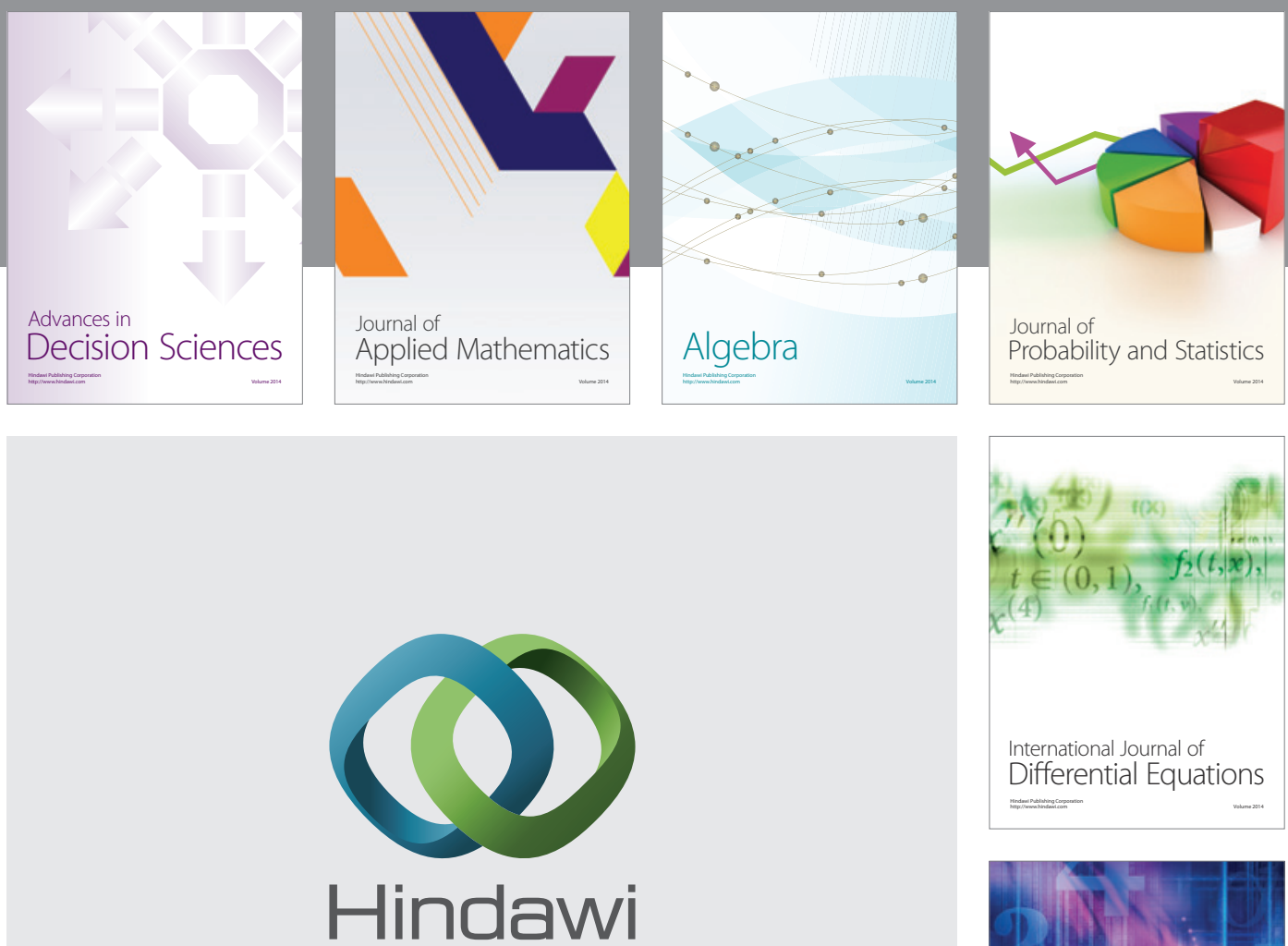

Submit your manuscripts at http://www.hindawi.com
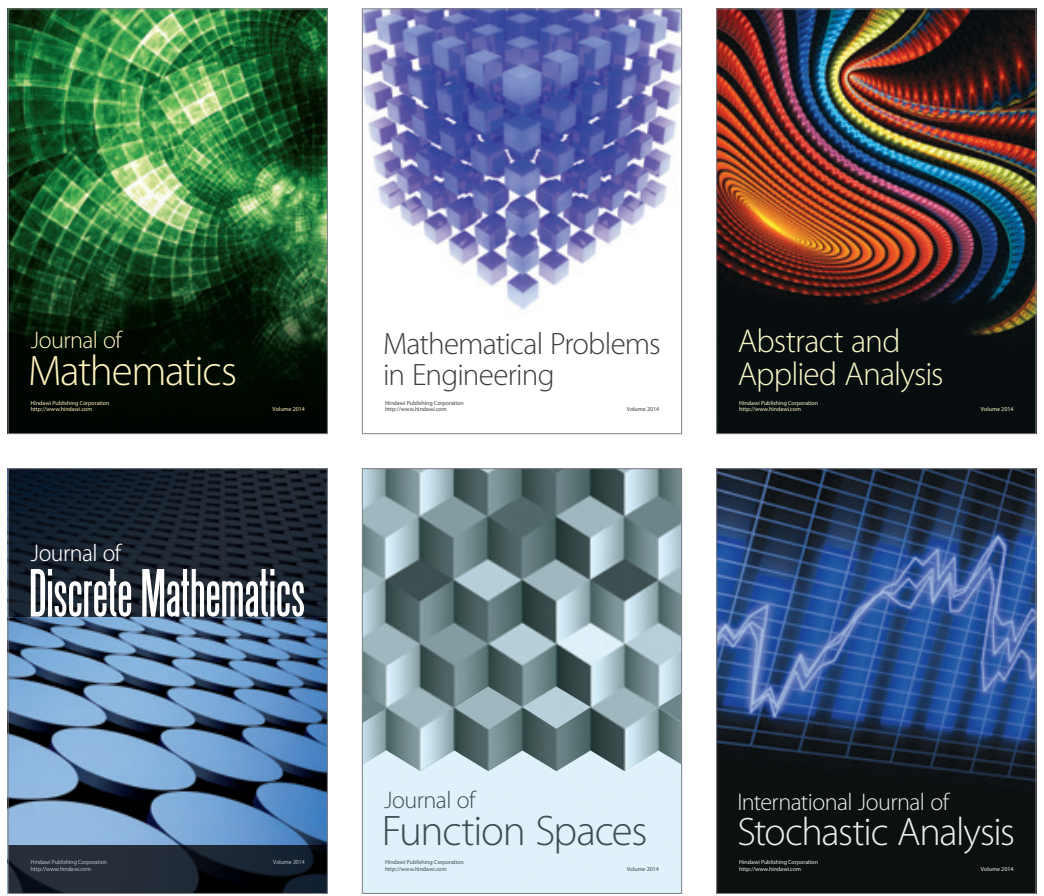

Journal of

Function Spaces

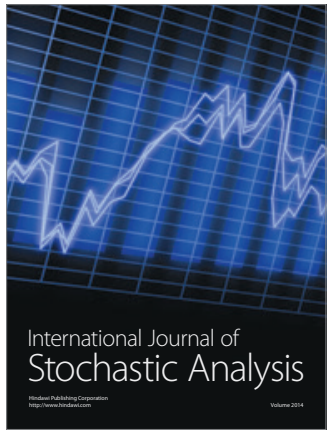

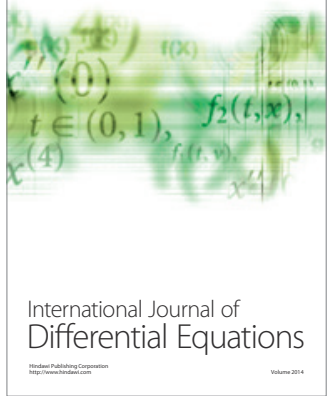
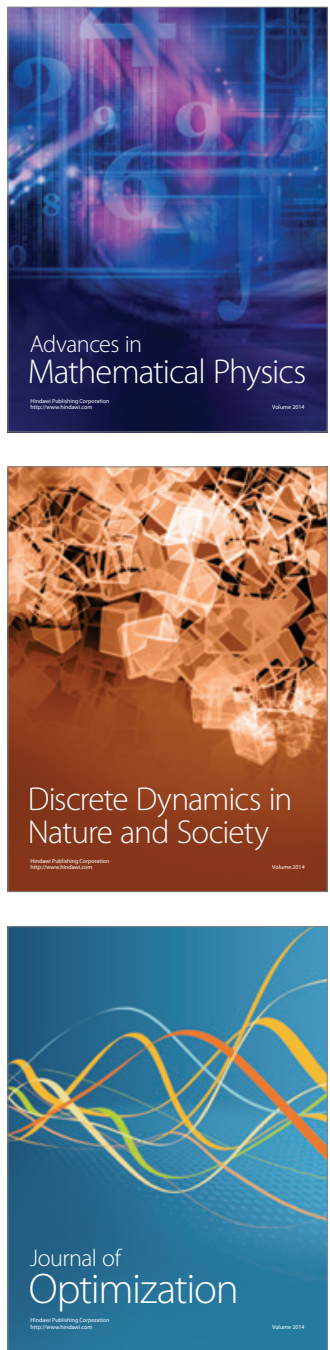